\title{
REVUE
}

\section{Les Chénopodiacées fourragères}

\author{
par M. G. CURASSON
}

Les Chénopodiacées (Salsolacées) des régions chaudes constituent en bien des endroits les « salt bushs ", ou buissons salés, qui croissent sur les terrains que les écologistes anglais et américains désignent sous le nom de salt-land, salt-steppe ou salt-desert; ces formations ont comme caractéristique de posséder une flore très pauvre de plantes à adaptations halophytiques, groupées en associations ouvertes. Ces formations sont répandues dans le Sud-Est de la Russie, la Perse, les Pampas de l'Argentine, les déserts de l'intérieur de l'Australie. L'Afrique du Nord, le Sahara jusqu'aux régions sahéliennes en possèdent aussi de grandes étendues. La nature du sol est telle qu'il est impropre à toutes les autres plantes.

Aussi, ces plantes ont-elles comme caractères dominants, l'adaptation à un sol riche en sels, leur résistance à la sécheresse; cela se traduit par un dóveloppoment radiculaire considérable; elles ont des feuilles entières, succulentes, avec un revêtement particulier qui parait velu.

Leur composilion les fait dédaigner des animaux quand ils ont à leur disposition d'autres plantes; mais quand ils ne trouvent qu'elles, ils les dévorent avidement.

Il est d'autres Chénopodiacées qui croissent ailleurs que sur les terrains salés; mais on les rencontre sur des sols pauvres, sablonneux, des déserts et subdéserts, én sorte que, comme les salt bushs, elles sont un apport nutritif important de ces régions, particulièrement en saison sèche. Certaines, comme les Atriplex, poussent sur des terrains très variés; leur multiplication se fait souvent par les animaux eux-mêmes, et leur capacité d'extension est considérable; cela s'est révélé au cours de la dernière guerre pour les Kochia, dont dos grainos venues accidentellement en Afrique nord-orientale avec les troupes australiennes ont été à l'origine de grandes étendues de Kochia, sur la nature desquelles on fut quelque temps indécis et sur lesquelles on fonda des espoirs peut-être prématurés.

Adaptées à des conditions de sol très variées, à la chaleur, à la sécheresse, les espèces, d'aspect varié, qui composent en partie la famille des Chénopodiacées devaient attirer l'attention des expérimentateurs à la recherche de plantes qui puissent constituer des terrains de parcours en élevage cxtensif, être à la rigucur cultivées pour la conservation et aussi être employées pour le maintien du sol. Les essais furent nombreux en Australie, en Afrique du Sud, en Amérique; plus timides en Afrique du Nord; au Soudan français, on réalisa une tentative non suivie. De ces recherches, il résulta que plusieurs Atriplex, Kochia, etc., méritaient d'être répandus dans leur zone normale d'habitat et parfois bien en dehors de celle-ci.

Mais, à mesure que se précisaient d'autres recherches concernant l'amélioration des pâturages subtropicaux et tropicaux, recherches qui faisaient entrer dans le cycle des essais, des graminées, des légumineuses, une défaveur atteignait- les Chénopodiacées. Il n'en reste pas moins qu'il est des conditions de sol et d'élevage dans lesquelles elles ne peuvent être remplacées.

Les travaux concernant les Salsolacées sont épars dans diverses publications de langue anglaise. La plupart ont été analysées dans "The use and misuse of shrubs and trees as fodder ", publié en 1947 par l' "Imperial bureau of pastures and field crops 》, auquel nous avons eu recours, ainsi qu'à des travaux plus récents.

\section{I. - PRINCIPALES ESPÈCES.}

\section{GenYe ATRIPLEX}

Ce genre comprend de nombreuses cspc̀cos, dont certaines ont une dispersion très étendue ; on en rencontre dans les diverses régions à terres salées du monde.

\section{Afrique du Nord :}

A. halimus existe dans le Sahara, le Soudan angloégyptien. C'est un arbuste de 1 à 2 mètres de hauteur; ses feuilles, à odeur caractéristique, sont broutées avec avidité par le mouton. Il est connu en Afrique du Sud sous le nom de $A$. capensis. 
Analyses de divers Atriplex.

\begin{tabular}{|c|c|c|c|c|c|c|c|}
\hline ESPËCES & PARTIES ANALYSÉES & EAU & $\begin{array}{l}\text { Protíist } \\
\text { brute }\end{array}$ & $\begin{array}{l}\text { LitruII } \\
\text { éthéré }\end{array}$ & $\begin{array}{c}\text { celltose } \\
\text { bruie }\end{array}$ & $\begin{array}{l}\text { IIIR:II } \\
\text { ncn } \\
\text { azoté }\end{array}$ & GEXDRES \\
\hline Atriplex capensis & Plante entière & 71,7 & 22,9 & 2,5 & 11,9 & 37,5 & 25,2 \\
\hline Atriplex halimoïdəs . ............ & Plante entière & 65,4 & 17,6 & 2,2 & 17,6 & 37,5 & 25,3 \\
\hline Atriplex nummularia. . & 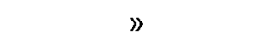 & 66,2 & 21,3 & 2,6 & 10,3 & 43,6 & 22,2 \\
\hline Atriplex prostrata..... & Plante en fleurs & $»$ & $.17,2$ & 1,7 & 10,5 & 45,5 & 25,1 \\
\hline Atriplex rhagodioïdes. & Feuilles & $"$ & 13 & 6 & 13,2 & 60 & $»$ \\
\hline Atriplex semibaccata. & Plante entière & 67,4 & 16,6 & 1,6 & 18,7 & 40,1 & 23,6 \\
\hline Atriplex tatarica ..... & $»$ & $»$ & 11,8 & 2,3 & 31,6 & 41,5 & 14,1 \\
\hline Atriplex vesicaria & Feuilles & $»$ & 11,4 & 2 & 14,2 & 46,1 & $\gg$ \\
\hline
\end{tabular}

A. mollis, du Sahara et Afrique du Nord, est surtout mangée par le chameau.

A. portulacö̈des est à vrai dire, une espèce méditerranéenne, considérée comme bon fourrage (Provence, Catalogne), dont l'introduction a été conseillée en Afrique du Nord.

\section{Afrique du Sud :}

A. albicans est un arbuste localisé dans une zone désertique proche de la mer.

A. capensis est un arbuste à feuilles succulentes, considéré comme le meilleur des "salt bushs " d'Afrique du Sud; il existe au milieu d'autres Chénopodiacées dans les sols pauvres du "brak veid ».

A. halimoides, buisson du Karroo, possède des feuilles semi-succulentes et poilues. Elle a été introduite dans d'autres régions.

A. nummularia et $A$. muelleri, surtout australiennes, existent aussi dans le Karroo.

\section{Amérique du Nord :}

A. canescens est une espèce très répandue sur les terres alcalines et salées. C'est un buisson atteignant jusqu'à 3 mètres, à feuilles cotonneuses. On le trouve au Texas Occidental, en Californie, au Nevada, etc. C'est un fourrage important, en raison de son abondance, du fait aussi qu'il est facilement accessible, volumineux, el que toutes ses parties sont recherchées des bovins, moutons et chèvres. Mange trop abondamment, il pourrait cependant produire des accidents.

A. confertifolia existe dans les zones désertiques salées; cet arbuste est parfois cultivé par semis.

$A$. coronata existe dans la zone aride de l'Arizona, ainsi que $A$. elegans, $A$. lentiformis, $A$. linearis, A. polycarpa.

A. nuttallii, espèce australienne de terrains salés, ne dépasse pas un pied de hauteur. C'est un bon fourrage dont l'étendue diminue en raison d'un pacage excessif.

\section{Amérique du Sud :}

Au Chili, on rencontre $A$. atacamensis, peu recherché; $A$. coquimbana, surtout mangé par les moutons, ainsi que $A$. deserticola, $A$. repanda. On a introduit, ainsi qu'au Brésil, $A$. semibaccata, $A$. nummularia, espèces australiennes.

\section{Asie :}

A. halimus, espèce africaine, se rencontre aussi en Syrie.

A. cana est une bonne espèce des subdéserts salés du Kazasktan.

A. pedunculata est rencontréa dans les zones subdésertiques de l'U.R.S.S.

A. stocksii est une bonne espèce pour le chameau dans l'Inde.

A. tatarica. A. verrucifera existent dans les régions désertiques orientales de l'U.R.S.S.

\section{Australie :}

A. angulata se rencontre dans l'État de Victoria, où on le considère comme un bon fourrage.

A. hymenotheca, de l'Ouest de l'Australie, a des feuilles bien appâtées.

A. leptocarpa est également un bon "salt bush» des mêmes régions.

$A$. muelleri est une espèce de valeur moyenne de l'État de Victoria.

A. prostrata se rencontre dans l'Ouest de l'Australie.

A. rhagodioïdes, dans l'État de Victoria, est classée parmi les meilleures espèces. On la retrouve dans I'Ouest de l'Australie.

A. semibaccata, dans les mêmes régions, a de bonnes feuilles. On l'a introduite dans diverses régions. Elle existe en Afrique du Sud, dans la région du Cap. 


\section{Genre BASSIA}

En Australie de l'Ouest, dans l'État de Victoria, les salt bushs de ce genre ne sont guère intéressants que lorsqu'ils sont jeunes; plus tard, ils forment de petits buissons bas, trop 'épineux pour être mangés; certains, comme $B$. birchii, deviennent alors fort gênants. On trouve $B$. anisacanthoides, $B$. convexula, $B$. echinopsila, $B$. lanicuspis, $B$. paradoxa, $B$. uniflora, cette dernière consideree comme bonne.

Dans l'Inde, $B$. latifolia est une bonne espèce, $B$. sedoïdes se rencontre dans les déserts asiatiques de I'U.R.S.S.

\section{Genre CAROXYLON}

Confondu parfois avec le genre Salsola, ce genre renferme plusieurs espèces sud-africaines. Leur composition moyenne est la suivante :

\begin{tabular}{|c|c|}
\hline Protéine brute & 23,45 \\
\hline Extrait éthéré . & 2,26 \\
\hline Cellulose brute. . & 10,03 \\
\hline Extrait non azoté & 46,61 \\
\hline Cendres $\ldots . . .$. & 17,65 \\
\hline $\mathrm{P}^{2} \mathrm{O}^{5} \ldots$ & 0,50 \\
\hline CaO... & 3,18 \\
\hline
\end{tabular}

C. articulatum, C. tetragonum, d'Afrique du Nord, sont mangées par le mouton et le chameau.

\section{Genre CHENOLEA}

C. tricornis est une bonne espèce australienne (Victoria). Dans le Sud Saharien, le Sahel soudanais, on rencontre $C$. canariensis.

\section{Genre CHENOPODIUM}

Les Chenopodium se rencontrent dans beaucoup de régions chaudes, particulièrement sur les terrains salés ou alcalins.

En Australie, diverses espèces buissonnantes ou simples herbes forment de bons pâturages, les meilleures étant $C h$. auricomum et $C h$. nitrariaceum. On trouve aussi Ch. album, Ch. triangulare (Nouvelles-Galles du Sud).

Ch. atriplicimum, Ch. carinatum sont mangées par le mouton et le chameau. La première pourrait causer des accidents au début de la végétation; la deuxième pourrait être cyanogénétique.

Plusieurs espèces sont mangées en Asie Orientale; en Asie Centrale, Ch. cubicum est assez bonne pour qu'on en ait tenté la culture.

En Amérique du Sud, Ch. quinoa, Ch. hircinum sont plutôt considérées comme de mauvaises herbes. La dernière peut envahir les luzernières.

\section{Genre Cornacula}

C. monacantha, espèce saharienne, est une des plantes préférées du chameau.

\section{Genre ENCHYLENA}

Plusieurs espèces australiennes sont connues sous le nom de "berry salt bushs 》, dénomination également attribuée aux Rhagodia. Ce sont des arbustes parfois utilisés pour la formation de haies. Certaines ont une bonne valeur fourragère, tel $E$. tomentosa que mange le bétail.

\section{Genre EXOMIS}

Exomis axyrioïdes est un salt bush fréquent en Afrique du Sud (Karroo) sur les terrains acides ou salés. C'est une plante recherchée du mouton.

\section{GenYe HALOCNEMUM}

Dans les zones subdésertiques de l'Afrique nordorientale, particulièrement en Tripolilaine, on rencontre abondamment $H$. strobilaceum, souvent associée à Haloxylon articulatum. On la retrouve sur les sols salés d'Asie Centrale. Sa composition est la suivante :

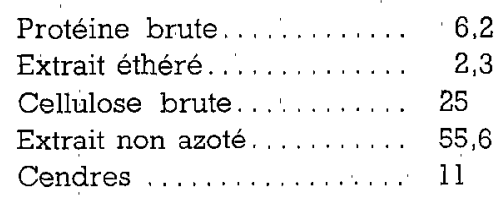

\section{GenYe HALOXYLON}

$H$ ammodendron, $H$. griffithii sont des buissons salés qui peuvent atteindre la taille d'arbres de l'Inde (Baloutchistan). On retrouve la première espèce en Asie Centrale, en Tripolitaine.

$H$. articulatum, $H$. persicum ( $H$. ammodendron) fournissent les, chameliers de l'Irak en nourriture pour leurs chameaux et en bois.

H. aphyllum, de l'Asie Centrale, est un grand arbuste sans valeur fourragère.

\section{Genre KOCHIA}

Ce genre renferme de nombreuses espèces qui constituent des "buissons salés " en diverses régions.

C'est surtout en. Australie du Sud (d'où d'ailleurs, on a exporté certaines espèces) qu'on les rencontre sous forme de buissons assez bas, de valeur diverse; on les connait sous le nom générique de "blue bushs »; assez forts en bois, ils portent de petites 
Analyses de diverses espèces de Kochia.

\begin{tabular}{|c|c|c|c|c|c|c|c|}
\hline ESPËCES & PARTIES ANALYSÉES & EAU & $\begin{array}{l}\text { Protillie } \\
\text { brute }\end{array}$ & $\begin{array}{l}\text { Extraltt } \\
\text { éthéré }\end{array}$ & $\begin{array}{c}\text { CLLLLLOSI } \\
\text { brute }\end{array}$ & $\begin{array}{c}\text { EXTR.IIT } \\
\text { non } \\
\text { azote }\end{array}$ & CLYRES \\
\hline Kochia brevifo:ia . . . . . . & Fouilles & $\gg$ & 24,8 & 2,5 & 11,1 & 33,5 & $»$ \\
\hline Kochia prostrata . . . . . . . . . . & Feuilles & $\gg$ & 9,8 & 3 & 35,3 & 39,2 & $》$ \\
\hline Kochia scoparia . & Graines & $\gg$ & 18,1 & 33,2 & 37,9 & 7,3 & 3,5 \\
\hline Kochia sedifolia . . . . . . . . . . . . . & Feuilles & $\gg$ & 17,2 & 2,6 & 22 & 41,5 & $\gg$ \\
\hline
\end{tabular}

feuilles charnues, cylindriques. Ils sont très résistants à la sécheresse; leur ordre d'appétence serait : $K$. planifolia, $K$. aphylla, $K$. sedifolia, $K$. pyramidata. Des accidents mortels auraient été observés chez des moutons ayant ingéré de grosses quantités du dernier.

Dans l'Ouest de l'Australie, on trouve $K$. brevifolia, $K$. villosa; au Queensland, $K$. coronata, $K$. georgei, $K$. triptera, $K$. tomentosa var. tenuifolia.

En Amérique du Nord (Utah) on trouve $K$. scoparia.

$K$. indica est une espèce de l'Inde que mange le chameau.

$K$.prostrata existe dans les déserts et semi-déserts asiatiques; elle est parfois cultivée.

\section{Genre PLINTHUS}

En Afrique du Sud, deux « buissons salés 》 du genre (très rapproché du genre Salsola et classés aussi dans la famille des Aizoacées) constituent de bons fourrages qui sont recherchés des moutons. Ce sont Pl. karooicus et PI. cryptocarpus.

\section{Gente RHäGODIA}

Diverses espèces de ce genre sont considérées en Australie, Nouvelle-Zélạnde, comme d'excellents fourrages. Les meilleures seraient : $R h$. parabolica,
$R h$. spinescens. Les autres sont : $R h$. gaudichaudiana, Rh. hostata, Rh. nutans.

\section{Genre SALICORNIA}

S. fruticosa est une plante des terrains salés d'Afrique du Nord, de Tripolitaine, d'Afrique du Sud. Les moutons et chèvres en sont friands.

$S$. foetida est une espèce de l'Inde.

\section{Genre SALSOLA}

Plantes des terrains salés (elles peuvent renfermer $40 \%$ de sel dans leur matière sèche), les Salsola soni représentées par de nombreuses espèces.

En Afrique du Sud : S. aphylla, S. canescens, S. glabrescens, $S$. nigrescens, $S$. tuberculata, S. zeyheri sont toutes mangées, surtout les formes courtes. Leur teneur en chlorure de sodium, parfois en nitrate, fait que malgré leur richesse relative en protéine, elles peuvent difficilement être mangées seules.

S. microphylla, S. tetragona sont des espèces sahariennes. De nombreuses espèces existent dans les zones salées des déserts et subdéserts asiatiques : $S$. arbuscula, $S$. brachiata, $S$. carinata, $S$. clavifolia, S. crassa, S. gemmascens, $S$. glabrescens, S. kali, S. rigida, S. subaphylla, S. turcomania, S. verrucosa.

Aralyses de divarses espàces de Salsola.

\begin{tabular}{|c|c|c|c|c|c|c|c|}
\hline ESPËCES & PARTIES ANALYSEEES & EAU & $\begin{array}{l}\text { Prutile } \\
\text { brute }\end{array}$ & $\begin{array}{l}\text { IrPRlll } \\
\text { éthéré }\end{array}$ & $\begin{array}{c}\text { CELUDLost } \\
\text { brute }\end{array}$ & $\begin{array}{l}\text { ETRLIT- } \\
\text { non } \\
\text { azoté }\end{array}$ & CENDRFS \\
\hline Salsola aphylla ..... . & Plante entière & 71,8 & 20 & 2 & 14,9 & 34,5 & 28,6 \\
\hline Salsola arbuscula . . . . & Début de floraison & » & 8,5 & 3 & 30,3 & 43,5 & 14,7 \\
\hline Salsola glabrescens.... & Plante entière & 58,6 & 17,9 & 1,9 & 15,6 & 48,7 & 15,8 \\
\hline Salsola nigrescens ........... & $\gg$ & $》$ & 10,6 & 1,2 & 28,3 & 42,8 & 17,1 \\
\hline Salsola rigida $\ldots \ldots \ldots \ldots$ & Début de floraison & 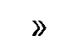 & 10,7 & 1,7 & 31,4 & 40,1 & 16,1 \\
\hline Salsola verrucosa . . . . . . . . . . & Plante entière & $\gg$ & 9,5 & 2,4 & 20,7 & 30,6 & 36,9 \\
\hline
\end{tabular}




\section{Genre SCHANGENIA}

S. baccata, en Asie mineure, est surtout mangée par le chameau.

\section{Genre SUIEDA}

S. fruticosa est une plante à chameau d'Afrique Orientale. En Asie on trouve S. maritima, également mangée par le chameau, comme $S$, microphylla. En Syrie, on signale $S$. monoïca, qu'on retrouve au Baloutchistan. La composition de S. maritima est la suivante :

$\begin{array}{lr}\text { Protéine brute } \ldots \ldots \ldots \ldots & 6,6 \\ \text { Extrait éthéré } \ldots \ldots \ldots \ldots & 2,8 \\ \text { Cellulose brute. } \ldots \ldots \ldots \ldots & 19,3 \\ \text { Extrait non azoté } \ldots \ldots \ldots \ldots & 59,2 \\ \text { Cendres } \ldots \ldots \ldots \ldots \ldots \ldots & 12,1\end{array}$

\section{II. - VALEUR ALIMENTAIRE - UTILISATION CULTURE.}

Les Chénopodiacées que nous venons d'énumérer ne constituent jamais des fourrages riches. Elles jouent cependant un rôle important dans l'alimentation des animaux, particulièrement du mouton, dans les zones où elles dominent et où, à certaines saisons, elles constituent à peu près la seule ressource.

Des essais pratiques réalisés en Australie et en Afrique du Sud ont montré que certaines peuvent rester, pendant de longues périodes, l'unique aliment. Ainsi, à la ferme expérimentale de Coolabals (Australie), des moutons ont vécu pondant vingt et un mois uniquement de salt bushs. En Afrique du Sud, un fermier a pu maintenir 300 moutons en bonne condition pendant deux mois sur un pâturage d'atriplex.

Les Salsolacées sont la plupart du temps consommées sur place par les animaux; mais on les a utilisées également, soit pour créer des pâturages, soit pour augmenter la résistance de pâturages herbacés, soit encore pour lutter contre l'érosion du sol.

Certaines d'entre elles se montrant particulièrement interessantes dans leur zone originelle, on a voulu les multiplier dans d'autres régions; on n'a pas toujours réussi. C'est que les plantes halophytes thermophiles et xérophiles ne s'adaptent pas forcément à un sol et un climat où elles ne retrouvent pas toutes les conditions qui les faisaient prospérer dans leur zone originelle.

Dans leur aire naturelle, la multiplication se fait facilement par les graines que rćpandent le vent ou les animaux; on peut cependant, avoir à les y multiplier parce que la pâture continuelle est un obstacle à leur dispersion. En Australie, on sème en poquets après avoir tracé quelques sillons. On peut aussi, là où elles ont tendance à disparaître, recourir au procédé qui réussit bien pour d'autres graines fragiles, et qui a réussi au Soudan français : on se borne à tracer quelques traits de charrue en saison sèche, dans le sens perpendiculaire aux vents dominants. Dans ces traits, s'accumulent les graines et un peu de terre, ce qui favorisera la germination à la saison des pluies. On a vu le procédé réussir au Soudan sur de véritables glacis où ne pouvaient, sans cela, s'arrêter les graines.

Les caractéristiques principales concernant leur composition sont la forto teneur en sels et en protéine. Quant à la cellulose, elle existe à des taux très variés ; cependant, la teneur est généralement assez faible, si on ne lient compte que des feuilles qui, le plus souvent, représentent la seule partie consommée. Il en résulte que certaines peuvent être comparées à d'autres aliments. C'est le cas pour Atriplex nummularia (cultivée) dont le foin a la composition suivante, comparée à celle du foin de luzerne.

\begin{tabular}{|c|c|c|c|c|c|c|}
\hline & Eall & Cendres & Proleine & Cellulose & $\begin{array}{c}\text { Matières } \\
\text { pon azolées } \\
\text { - }\end{array}$ & $\begin{array}{l}\text { Maticres } \\
\text { grasses } \\
-\end{array}$ \\
\hline & 10 & 7,82 & 4,11 & 1,01 & 10,71 & 0,56 \\
\hline Luzerne. ... . . . . & 10,95 & 1,80 & 4,91 & 6,34 & 11,09 & 0,86 \\
\hline
\end{tabular}

La teneur en protéine et en matières minérales fait que, dans les pâturages de saison sèche, l'association des "salt bushs 》 ou des "blue bushs " avcc les herbes sèches qui conservent une certaine proportion d'hydrates de carbone assure une ration assez équilibrée pour permettre aux animaux un entretien assez long.

Voici maintenant quelques espèces qui ont été soumises à. la multiplication.

Atriplex nummularia (Old man salt bush), c'est l'espèce la plus appréciée pour la culture. Elle est très répandue en Australie; en Afrique australe, on l'a répandue dans toutes les zones sèches. On l'a introduite au Soudan, mais elle ne s'y est pas répandue. C'est la forme buissonnante qu'on cultive comme plante de haie; elle résiste très bien à la sécheresse, et est volontiers mangée par toute sorte de bétail. Elle forme un arbuste ramifié, touffu, avec un feuillage argenté et charnu, qui lui donne un aspect particulier.

Ce qui linite soin extension à des nouvelles zones, c'cot qu'il ne se reproduit pas partout de graines. Ainsi, en Californie, il ne donne pas de-graines; de même au Soudan français; il faut alors reproduire par boutures ou éclats de racines.

Là où la plante se reproduit par graincs, la moilicure façon d'opérer est de semer en pépinière, de transplanter les jeunes plants quand ils ont atteirnt à peu près 30 centimètres; le semis direct des graines dans les champs (soit à la volée, suit par semis en sillons) donne raroment dos rosultats setisfaisents. 
Les graines de bonne qualité germent dans les pépinières en dix jours à peu près, et la plante est assez grande pour être transportée au bout de douze mois. La graine est assez exigeante. et demande un sol bien préparé, ombragé. On plante à 4 mètres environ dans tous les sens.

I,a multiplication se fait bien, hors I'habitat de la plante, à l'aide de boutures de tête herbacées qu'jl faus abriter un peu et arroser quelques jours. On profitera de la saison des pluies; on peut multiplier aussi par marcoitos. De toute façon, il ne peut pas faire pâturer avant un an

Atriplex semibaccata (Creeping salt bush), originaire d'Australie, a été introduit en Afrique du Sud, aux îles Hawaï; en Californie, il a mieux réussi que le précédent; par contre, il n'a pas réussi au Soudan français; il s'est facilement répandu en Afrique australe. Il est cependant plus exigeant que $A$. nummularia au point de vue humidité. On peut lui reprocher d'être un «buisson rampant ». Il se couche en effet sur le sol, ce qui le livre entièrement à la dent des animaux alors que $A$. nummularia, qui atteint 3 à 4 mètres, leur échappe en partie.

Dans l'Australie de l'Ouest, on prolonge la durée des pâturages composés de graminées annuelles en y incorporant des "salt bushs », particulièrement A. semibaccata (et aussi A. leptocarpa, Kochia brevifolia). Ces buissons saiés donnent de la stabilité au pâturage; il semble aussi qu'ils gênent la pousse des graminées au profit des légumineuses du pâturage.

Atriplex halimus, le "guettaf» des Arabes (qu'on retrouve en France dans la région méditerranéenne), est très répandu en certaines régions d'Afrique du Nord, dans les zones sèches et pauvres. Il a une rusticité telle qu'une branche abandonnée sur le sol donne souvent naissance à un pied nouveau au printemps; aussi la multiplication est-elle facile par boutures ou éclats de souche, plus difficile par semis.

Atriplex lentiformis a été répandu dans toutes les zones désertiques du centre de l'Amérique. Il est particulièrement intéressant en raison de sa taille (2 à $3 \mathrm{~m}$.) et de l'abondance de son feuillage. Il est très recherché du bétail.

Atriplex halimoïdes, $A$, campanulata, $A$. vesicaria, espèces australiennes, ont été essayées avec plus ou moins de chances, en d'autres régions. A. halimoïdes a été introduit sans grand succès en Afrique du Nord. Des graines importées au Soudan français se sont révélèes stériles.

Salsola spp. En Afrique du Sud, on a tenté la culture de diverses Salsola; mais ces plantes privent rapidement le sol de ses sels (leur teneur en phosphore, en hiver, peut dépasser $0,25 \%$ ). Cette teneur en phosphore explique que sa présence dans un pâturage naturel est considérée, au Bechuanaland, comme le signe qu'il n'y a pas de "lamsiekte » c'est-à-dire de maladie par carence phosphorée.

Salsola Kali peut être utilisée comme foin et comme ensilage, ce dernier préparé avec la plante et de la mélasse, ou avec de la luzerne. La protéine digestible est comparable à celle du foin de trèfle. et l'ensemble des éléments nutritifs égale ceux de la paille d'avoine. Cependant, le foin n'est bien acceptć des moutons que si on l'humecte avec addition de mélasse. On a pu l'utiliser ainsi pendant quatre périodes hivernales, chez des brebis, sans effet nocif.

Salsola rickteri, introduite du Turkestan au Sahara, pourrait fournir à la fois du bois et du fourrage.

Chenopodium urbicum, $C h$. album ont été cultivées en Asie centrale dans les zones subdésertiques. On peut les ensiler. La composition de $C h$. urbicum est la suivante :

\begin{tabular}{|c|c|}
\hline Protéine brute & 20,3 \\
\hline Extrait éthéré ... & 2,3 \\
\hline & 21, \\
\hline & \\
\hline & \\
\hline
\end{tabular}

Chenopodium quinoa est cultivée largement. La graine sert à faire une sorte de, pair, ou est distribuée aux volailles et au bétail. Elle renferme $57 \%$ de matières non azotées, $23 \%$ de matières albuminoides.

Kochia prostrata a été cultivée dạs les sols sablonneux et salés de l'U.R.S.S. ; elle se développe bien par semis.

Kochia brevifolia, bien que moins recherchee des animaux que d'autres Kochia, a été répandue en Australie à la fois pour lutter contre l'érosion du sol et pour assurer la stabilité des pâturages herbacés.

Kochia sedifolia a été introduite au Soudan français en 1926, dans la zone sahélienne (El Oualadji). Les semis irrigués ont permis un repiquage et des plantes ont pu prospérer. La tentative n'a pas été poursuivie.

Kochia villosa n'a pas donné de résultats intéressants en Afrique du Nord.

Suæda fruticosa a été cultivée (par semis) au Soudan anglo-égyptien. 